\title{
DinoApp: aplicativo para apoio ao tratamento de crianças com câncer
}

\author{
João Pedro Silveira e Silva ${ }^{1}$, Mayra Camargo Cademartori ${ }^{1}$, Victória de Avelar Duarte ${ }^{1}$, \\ Mariana Bohns Michalowski ${ }^{2}$, Érika Cota ${ }^{1}$ \\ ${ }^{1}$ PET Computação - Instituto de Informática \\ ${ }^{2}$ Hospital de Clínicas de Porto Alegre \\ Universidade Federal do Rio Grande do Sul (UFRGS) \\ Porto Alegre - RS - Brazil \\ \{joao.silva, mayra.cademartori,victoria.duarte, erika\}einf.ufrgs.br \\ mmichalowski@hcpa.edu.br
}

\begin{abstract}
This paper presents DinoApp, a mobile application developed to support the medical care of children with cancer. DinoApp brings information about the disease and the treatment. For the children, this information is presented in a playful way. The app also offers a communication channel to the medical team and an agenda for the scheduling of medical procedures in the post hospitalization period. With DinoApp we aim at increasing the patient engagement during pediatric cancer treatment.
\end{abstract}

Resumo. Este artigo apresenta o DinoApp, um aplicativo mobile desenvolvido para apoiar o atendimento médico de crianças com câncer. $O$ DinoApp leva informações sobre a doença e o tratamento. Para as crianças, essas informações são apresentadas de forma lúdica. O aplicativo também oferece um canal de comunicação entre a família e a equipe médica, além de uma agenda para o controle de procedimentos médicos no período pós-internação. O DinoApp é um protótipo desenvolvido para ajudar a aumentar o engajamento do paciente durante o tratamento do câncer pediátrico.

\section{Introdução}

Segundo o Ministério da Saúdẹ, a taxa de cura das neoplasias na infância no Brasil é da ordem de $60 \%$, um valor baixo quando comparado aos países desenvolvidos. Dentre as várias possíveis razões (sociais, individuais, médicas), as dificuldades pessoais e familiares são frequentemente observadas na prática clínica. O tratamento, que dura em média 18 meses, acontece tanto no ambiente hospitalar quanto domiciliar. No ambiente hospitalar, paciente e familiares contam com todo o suporte para os cuidados e procedimentos do tratamento. Já no ambiente doméstico, o distanciamento físico da equipe de saúde dificulta esse atendimento. Assim, a família muitas vezes acumula dúvidas, inseguranças, crenças ou mesmo comportamentos que acabam se refletindo nos procedimentos necessários ao tratamento e diminuindo a adesão ao mesmo.

Uma revisão sistemática e meta-analítica [Mao and et al. 2020] enfatizou que a combinação da intervenção Health com a gestão profissional é fundamental para atingir

\footnotetext{
${ }^{1}$ http://www1.inca.gov.br/wcm/incidencia/2017/pdf/versao-completa.pdf
} 
a eficácia clínica ideal. Este artigo apresenta o DinoApp, uma solução móvel que tem o objetivo de aproximar a equipe de saúde do paciente em tratamento domiciliar pósinternação. O aplicativo é focado em crianças da faixa etária de 5-6 anos e seus familiares e busca responder às principais dificuldades de adesão ao tratamento identificadas por uma equipe de saúde multidisciplinar do setor de oncopediatria do Hospital de Clínicas de Porto Alegre (HCPA).

O artigo está organizado da seguinte maneira: a Seção 2 descreve o processo de entendimento e especificação do DinoApp. A Seção 3 apresenta a arquitetura da solução e os aspectos mais importantes da implementação e validação da solução. A Seção 4 conclui o artigo.

\section{Processo de entendimento e especificação do DinoApp}

Foi feita uma pesquisa com pacientes, familiares e a equipe de saúde (médicos, nutricionistas, psicólogas, enfermeiras, serviço social, farmacêuticos, pedagogos e recreação) para levantamento de interesses e dificuldades enfrentadas ao longo do tratamento. A partir disso, levantou-se a necessidade de aproximação da equipe de saúde e da família no período pós-internação. Definiu-se, ainda, a hipótese de que um aplicativo poderia suprir essa necessidade. Assim, a parceria entre o HCPA e o PET Computação UFRGS teve como objetivo o desenvolvimento de um aplicativo que permitisse validar a hipótese levantada.

Um workshop de lean inception foi feito com uma equipe multidisciplinar composta pela equipe de saúde e desenvolvedores membros PET. Como resultado, alinhou-se a visão do produto (Figura 1) e definiram-se as funcionalidades que deveriam fazer parte do MVP. Definiu-se, ainda, o nome e identidade da solução, que remete ao personagem referência do setor de oncopediatria do HCPA, um dinossauro que fica na entrada desta ala do hospital.

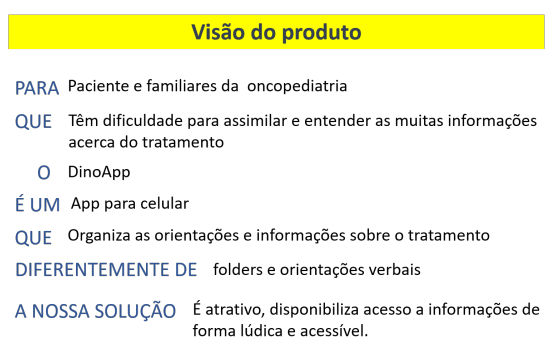

(a)

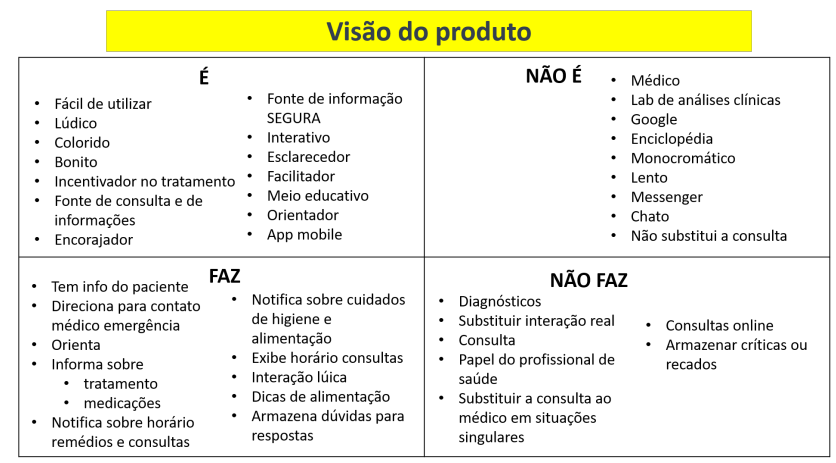

(b)

Figura 1. Visão do produto derivada do workshop de inception

\subsection{Mínimo Produto Viável}

Foram planejadas as seguintes funcionalidades para o MVP:

- Calendário: marca consultas e horários de medicamentos, fornece lembretes e auxilia na organização de pais e responsáveis. 
- Contatos: lista de contatos e telefones do hospital e/ou da equipe de saúde relevantes para o paciente.

- Glossário: verbetes em linguagem acessível, feitos por profissionais da área, de termos técnicos usados durante tratamentos.

- FAQ: área de perguntas-e-respostas frequentes dos pacientes e de seus responsáveis, na qual podem submeter suas próprias dúvidas a serem adicionadas à seção do tratamento correspondente.

- Notas: área pessoal para anotações e lembretes contextualizada a seções de consulta com os médicos responsáveis do paciente. Livre para edição do usuário.

- Jogos: área infantil, na qual o paciente irá encontrar jogos educativos ou para simples entretenimento.

\section{Projeto e Implementação}

Optou-se pelo desenvolvimento de um app híbrido usando tecnologia PWA, pela sua flexibilidade, suporte e facilidade de aprendizado, considerando o contexto de alta rotatividade do time de desenvolvimento.

Um PWA é uma aplicação Web que se comporta essencialmente como um aplicativo nativo, quando há a possibilidade de ser instalado.

Para complementar o aplicativo definiu-se ainda pela criação de uma Web API RESTful, responsável pelo fluxo, segurança, sincronia e armazenamento a longo-prazo de dados relevantes.

\subsection{Arquitetura}

O desenvolvimento segue um processo ágil e a arquitetura planejada pode ser vista na Figura 2. A solução se baseia em dois pacotes principais que representam o aplicativo do usuário (App - PWA) e a aplicação do servidor (Web API).

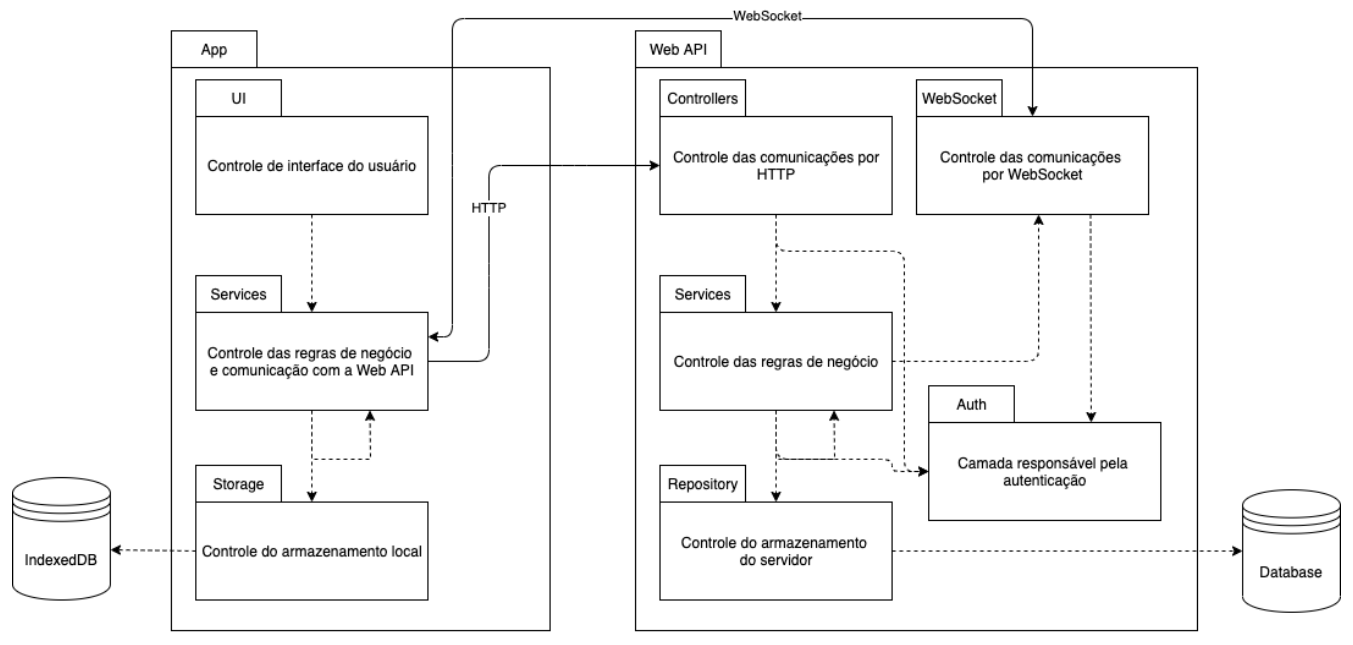

Figura 2. Arquitetura do Aplicativo

A Web API é dividida em 3 camadas essenciais: controllers, services e repositories, também utilizando-se da framework Spring para Java.

A camada de UI (User Interface) do aplicativo web utilizou a biblioteca ReactJS, sendo responsável por renderizar a interface com o usuário. Services em ambos os pacotes 
são responsáveis por lidar com regras de negócio. As camadas de Storage no PWA e Repository na Web API são responsáveis por lidar com o armazenamento de entidades. Controllers são responsáveis por receber requisições HTTP, enquanto WebSocket envia e recebe requisições do tipo WebSocket que abre um canal de comunicação servidor-cliente possibilitando a sincronização de dados em tempo real. Auth é responsável pelo processo de autenticação e autorização do usuário através do serviço de identidade do Google. Por requisitos de segurança toda informação provinda do aplicativo é considerada como insegura, sendo necessárias validações também por parte do servidor.

\subsection{Implementação e Validação}

O app prevê dois perfis de usuários: equipe de saúde (ES) e responsáveis por pacientes (RP). O perfil ES tem acesso às funcionalidades de edição do glossário, FAQ (criar novas entradas e responder a novas perguntas feitas pelas famílias) e lista de contatos disponível para cada paciente. O perfil RP pode editar a agenda (inclusive com a possibilidade de sincronização opcional com dados do Google), pode criar e editar anotações, pode inserir novas perguntas na FAQ e tem acesso à área infantil do app. Na área infantil, foram desenvolvidos até o momento cinco jogos dentro da arquitetura proposta. Toda a estrutura, servidor e softwares seguem a LGPD.

Todas as funcionalidades previstas no MVP foram implementadas, com exceção da agenda, que está em fase de desenvolvimento com previsão de entrega para julho/21. Atualmente, estamos iniciando a etapa de validação do DinoApp junto à equipe de saúde. A segunda etapa de validação será feita com um conjunto de usuários selecionados. Essas validações se referem ao uso do aplicativo em si. Após essa etapa, que tem previsão de entrega para outubro/21, o app poderá ser disponibilizado para os pacientes do HCPA no contexto de um estudo específico que deverá avaliar o impacto do seu uso no engajamento dos pacientes ao tratamento.

\section{Discussão}

Este artigo apresentou o DinoApp, um app de suporte ao tratamento do câncer infantil. O aplicativo é uma iniciativa conjunta do HCPA e do PET Computação UFRGS e tem o objetivo de aproximar a equipe médica do paciente e cuidadores, buscando aumentar o engajamento ao tratamento.

Soluções similares como Wecancer ${ }^{2}$ e Breast Cancer Healthline ${ }^{3}$ não são focados no paciente pediátrico. Além disso, são soluções independentes, enquanto o DinoApp propõe-se a facilitar a comunicação com a equipe médica do paciente.

$\mathrm{Na}$ etapa de validação com os pacientes, além das questões de usabilidade e funcionalidade do app, pretende-se avaliar também as questões de comunicação entre paciente/cuidadores e equipe médica e eventuais impactos desta nova forma de comunicação.

\section{Referências}

Mao, Y. and et al. (2020). Impact and efficacy of mobile health intervention in the management of diabetes and hypertension: a systematic review and meta-analysis. BMJ Open Diabetes Research and Care, 8(1):e001225. DOI:10.1136/bmjdrc-2020-001225.

\footnotetext{
${ }^{2}$ https://wecancer.com.br/

${ }^{3}$ https://play.google.com/store/apps/details?id=com.healthline.breastcancer
} 